\title{
Improving Laboratory Assessment in Disorders of Sex Development through a Multidisciplinary Network
}

\author{
Sabine E. Hannema ${ }^{a, c}$ Yolanda B. de Rijke ${ }^{b}$

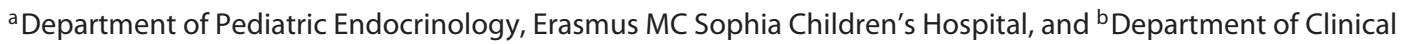 \\ Chemistry, Erasmus MC, University Medical Centre Rotterdam, Rotterdam, and ' Department of Paediatrics, Leiden \\ University Medical Centre, Leiden, The Netherlands
}

\section{Keywords}

Diagnostic process - Disorder of sex development . European Reference Network - Laboratory protocol . Rare endocrine disorders

\begin{abstract}
The aim of the European Reference Network for Rare Endocrine Disorders (Endo-ERN) is to ensure equal access to highquality care for all those affected by a rare endocrine condition across Europe, such as a disorder/difference of sex development (DSD), both for children and adults. Although differences in resources, health care systems, and health insurances between the European countries are challenging and require political action, a European laboratory network within Endo-ERN could improve the diagnostic process in individuals with DSD, building on the work done by previous European collaborations such as the COST action DSDnet. In close collaboration, clinicians and laboratory specialists must make every effort to standardize diagnostic protocols, achieve necessary harmonization of various laboratory tests, e.g., the hCG stimulation test, and implement an external quality control system. This should ideally result in comparable quality across the network centers allowing the sharing of reference values. This would not only improve patient care but also greatly facilitate research. @ 2018 The Author(s)

Published by S. Karger AG, Basel
\end{abstract}

\begin{tabular}{ll}
\hline KARGER & $\begin{array}{l}\text { ( } 2018 \text { The Author(s) } \\
\text { Published by S. Karger AG, Basel }\end{array}$ \\
$\begin{array}{l}\text { E-Mail karger@karger.com } \\
\text { www.karger.com/sxd }\end{array}$ & $\begin{array}{l}\text { Karger } \\
\text { This article is licensed under the Creative Commons Attribution- } \\
\text { NonCommercial-NoDerivatives 4.0 International License (CC BY- } \\
\text { NC-ND) (http://www.karger.com/Services/OpenAccessicense). } \\
\text { Usage and distribution for commercial purposes as well as any dis- } \\
\text { tribution of modified material requires written permission. }\end{array}$
\end{tabular}

Disorders/differences of sex development (DSD) are generally rare conditions requiring specialized care by multidisciplinary teams. Recently, the European Union has launched an initiative to establish European Reference Networks (ERNs) for rare disorders. The aim of these networks is to ensure equal access to high-quality care for all those affected by a rare condition across Europe, both children and adults. The largest network that has successfully applied and has recently become operational is the ERN Rare Endocrine Disorders (Endo-ERN). Within this ERN, several main thematic groups have been formed, one of which is "Sexual Development \& Maturation," which includes DSD.

The work-plan of the Endo-ERN has defined several work packages, one of which is "Diagnostics \& Laboratory Analysis." Among the tasks of this work package are the creation of an EU network of specialized accredited laboratories that offer diagnostic tests for endocrine diseases and the development of a web-based external quality control application. Concerning laboratory determinations relevant to DSD, this laboratory network within Endo-ERN can build on the work done within the COST Action "DSDnet" by the working group "Harmonization of Laboratory Assessment” (www.dsdnet.eu). This working group has recently published a position paper on steroid hormone analysis in diagnosis and treatment of DSD

Sabine E. Hannema

Erasmus MC - Sophia Children's Hospital Sp-3435 Postbus 2060

NL-3000 CB Rotterdam (The Netherlands)

E-Mail s.hannema@erasmusmc.nl 
[Kulle et al., 2017] and has established a pilot external quality assurance (EQA) program for serum dihydrotestosterone (DHT) [Greaves et al., 2017].

Laboratory investigations play a key role in the diagnostic workflow in individuals with suspected DSD [Hughes et al., 2006]. Establishing the right diagnosis is essential to counsel on prognosis, appropriate therapy, and necessary screening for associated comorbidities, and, especially in neonates, on the sex of rearing. In the last decade, next-generation sequencing has become widely available, and consequently the introduction of gene panels has made it possible to analyze a large number of genes in a short period of time. Besides genetic testing, endocrine investigations are still needed. Gene mutations can lead to a highly variable phenotype. For example, NR5A1 mutations in 46,XY individuals can result in phenotypes ranging from complete gonadal dysgenesis to male infertility [Achermann et al., 1999; Bashamboo et al., 2010]. Thus, hormone measurements remain essential for the assessment of gonadal function. Furthermore, when unclassified variants are found with genetic testing, hormone measurements can also be helpful to assess the likelihood that these variants are pathogenic. For example, if variants are found in the gene encoding $17-\beta$-HSD type 3 , a high androstenedione/testosterone ratio in the hCG test supports pathogenicity. In addition, measurement of various hormones is helpful in monitoring of spontaneous pubertal development and of hormonal therapy [Bhasin et al., 2010; Speiser et al., 2010]. Ideally, all tests considered essential for diagnostic purposes and for monitoring of therapy should be available and of sufficient quality across Europe.

\section{Selection of Hormone Measurements Essential for Diagnosis and Management of DSD}

Consensus amongst clinicians is required on what tests are considered essential in the diagnosis and management of DSD. The consensus statement from 2006 suggests 17- $\alpha$-hydroxyprogesterone (17OHP), testosterone, gonadotropins, anti-müllerian hormone (AMH), serum electrolytes, and urine analysis as the set of initial investigations when DSD is suspected, which may be followed by further investigations such as an hCG test, ACTH test, and urinary steroid profile. In 2011, the Society for Endocrinology UK has provided guidance on the initial investigation of a child with DSD which has been updated in 2015 [Ahmed et al., 2011, 2016]. Their first line of recommended endocrine laboratory investigations includes $17 \mathrm{OHP}$ to exclude congenital adrenal hyperplasia (CAH). If $\mathrm{CAH}$ is suspected, androstenedione, testosterone, possibly renin, and a urinary steroid profile can be measured. In children with a karyotype other than 46, XX, measurement of AMH and an hCG test is suggested to investigate testicular development and androgen synthesis. At a minimum, testosterone, androstenedione and DHT should be measured in the hCG test [Ahmed et al., 2016]. If testosterone does not rise sufficiently after hCG stimulation, a short ACTH stimulation test should be considered [Ahmed et al., 2016].

A survey among various European DSD clinics has shown considerable variation in the choice of diagnostic tests. This may partly be due to nonavailability of tests in certain clinics but might also be related to different protocols being used to establish a diagnosis. It would be helpful to develop a European guideline describing the diagnostic work-up of DSD. Depending on the differential diagnosis and age of the child, a different set of tests may be needed [Hughes et al., 2006]. A recommended diagnostic workflow should be described for several fairly common scenarios such as the neonate with ambiguous genitalia or the adolescent presenting with absent or abnormal pubertal development [Ahmed et al., 2011, 2016].

That steroid hormone measurements play an essential role in the monitoring of therapy is clear from various guidelines. Measurement of 17OHP, androstenedione, and testosterone is recommended to assess the adequacy of glucocorticoid therapy in CAH [Speiser et al., 2010], and measurement of testosterone levels is recommended in men receiving testosterone therapy because of androgen deficiency [Bhasin et al., 2010].

The availability of a predefined diagnostic panel considered essential for standardized evaluation and followup of individuals with DSD should be a requirement for all DSD expert centers just as the presence of a multidisciplinary DSD team [Ahmed et al., 2016].

\section{Performance of Hormone Measurements}

The quality assessment of the endocrine tests, dependent on the right pre-analytical, analytical, and post-analytical conditions, is considered essential for diagnosis and management of DSD and should be regularly monitored by an (inter)national EQA program.
136

Sex Dev 2018;12:135-139

DOI: $10.1159 / 000486329$
Hannema/de Rijke 
Timing of Laboratory Investigations and Test

\section{Protocols}

During the diagnostic workflow of a child with DSD, optimal timing of hormone measurements is important to produce reliable and useful results, and it may be necessary to repeat investigations at a later age [Ahmed et al., 2016]. While the measurement of adrenal steroids to diagnose CAH may be less reliable during the first $36 \mathrm{~h}$ of life, a urinary steroid profile cannot be used to assess the likelihood of $5 a$-reductase deficiency before 3 months of age [Ahmed et al., 2016]. The hypothalamus-pituitarygonadal (HPG) axis is active prenatally, during minipuberty, with peak testosterone levels at 1-3 months of age in boys [Kuiri-Hanninen et al., 2014] and then from puberty onwards. In the periods where this axis is quiescent, basal steroid measurements are uninformative so that a stimulation test is needed.

The hCG test is commonly used in the initial evaluation of a child with 46,XY DSD, but this test is not standardized. Protocols range from a single injection to 3 injections on consecutive days, to 6 injections every other day, to more extended stimulation for up to 3 weeks. Furthermore, there is a range in dosing from 500 to $1500 \mathrm{IU}$ or a weight or body surface area based dose. Blood samples for steroid measurements are drawn 24-72 h after the (last) injection [Kolon and Miller, 2001; Feyaerts et al., 2002; Ahmed et al., 2011]. There is a need for standardization of the test protocol to ensure that test results are comparable across different European countries. The protocols may need to be different for various age groups as the gonad is more responsive to hCG during periods when the HPG axis is active, i.e., the first half year of life (minipuberty) [Kuiri-Hanninen et al., 2014] and puberty, than in childhood when the HPG axis is quiescent [Bhowmick and Gidvani, 2000].

\section{Laboratory Analysis of Steroids}

Accurate measurements of steroid concentrations are critical to support clinical decisions. However, in neonates, measurement of steroids may be problematic. Besides quite different concentrations of many steroids in neonates' serum compared to those in older children, neonatal serum contains a different mix of steroids produced by the fetal adrenal zone that interferes with $17 \mathrm{OHP}$, androstenedione, and testosterone measurement [Wong et al., 1992; Wudy et al., 1995]. In addition, interfering molecules can also be drugs or structurally related endogenous compounds, e.g., DHEAS interferes with testosterone [Warner et al., 2006; Middle, 2007] and methylprednisolone with cortisol immunoassays. Fur-

Improving Laboratory Assessment in DSD thermore, nonspecific cross-reactivity leads to falsely elevated steroid concentrations.

To improve sensitivity and specificity, an increasing number of clinical laboratories use LC-MS/MS (tandem liquid chromatography-tandem mass spectrometry) methods for steroid hormone measurements. Furthermore, the LC-MS/MS method facilitates steroid profiling which is very informative in distinguishing almost all steroid related disorders. Although costs and required technical skills have limited the adoption of this state-of-theart technology in all regions of Europe, this method for steroid analysis is becoming increasingly available for routine use. Of the hormones essential for the diagnosis and management of DSD, reference LC-MS/MS method procedures have only been developed for cortisol and testosterone. These methods have been deployed to produce higher-order certified reference materials which are used to calibrate commercial immunoassays generally used in routine clinical laboratories. Still, the problem with the standardization of both automated immunoassays and radio- or enzyme-linked immunoassays for steroids remains.

Laboratories should aim to participate in activities of peer comparison such as a sample exchange or preferably when available subscribe to an EQA program to improve quality and comparability between laboratory tests. The availability of certified reference materials, reference methods, reference laboratories, and reference intervals and decision limits will improve the standardization of steroid analysis. In addition to standardization, reference intervals and decision limits are of tremendous value for the correct interpretation of steroid concentrations in premature and term neonates. However, reference intervals are not widely available, and in contrast to earlier anticipation these are mostly dependent on individual specific laboratory settings determined by sample workup and/or instrumentation. It has become difficult to recruit healthy children, especially neonates or premature infants, to establish normative reference data from a control cohort. This is mainly the result of ethical concerns and prevents the implementation of accurate age-, Tanner stage- and sex-specific reference intervals. A broad European initiative is needed to make good reference values for steroids available to clinical laboratories.

To further reduce the variability and interferences in steroid measurements, which can affect patient diagnosis and management, initiatives to support harmonization have been embraced globally. The working group "Harmonization of Laboratory Assessment" of the COST Action "DSDnet" has already started to work on an EQA

Sex Dev 2018;12:135-139 DOI: $10.1159 / 000486329$ 
program for 17OHP [Kulle et al., 2017]. Recently, a first EQA program for the harmonization of serum dihydrotestosterone measurement has been launched [Greaves et al., 2017]. The CDC established the Hormone Standardization Program to improve the accuracy of testosterone assays (https://www.cdc.gov/labstandards/pdf/ hs/Testosterone_Protocol.pdf). An important aspect of harmonized reference intervals is the development of criteria for a laboratory to use a common reference interval.

Current guidelines use absolute decision limits, such as aiming for a serum testosterone concentration of 14.1$24.5 \mathrm{nmol} / \mathrm{L}$ midway between injections in men being treated with testosterone [Bhasin et al., 2010], or rejecting the diagnosis $\mathrm{CAH}$ when ACTH-stimulated 17OHP is $<50 \mathrm{nmol} / \mathrm{L}$ [Speiser et al., 2010] although cut-offs may differ depending on the assay. International harmonization of assays and the use of common reference intervals would allow clinicians to use cut-offs from guidelines with confidence that the decision limits are applicable to their local situation. Ensuring that hormone measurements are directly comparable across countries would not only be of clinical value but would also greatly facilitate international research that relies on steroid measurements. These benefits justify investing in international rather than national harmonization.

\section{Availability of Essential Tests}

Not all tests that are considered necessary for the diagnostic evaluation and monitoring of treatment in individuals with DSD are currently locally available at all centers delivering specialist care for individuals with DSD as was shown in a recent survey amongst European and non-European centers [Kyriakou et al., 2016]. Essential diagnostic hormone tests that were frequently reported to be unavailable were AMH (21\%) and DHT (19\%). Although samples can be sent to a laboratory elsewhere, this will result in longer turnaround times. If a test was not available at a local accredited laboratory, clinicians were less likely to routinely perform this test. Most clinicians to whom these tests were unavailable indicated they would consider performing the test if it was available [Kyriakou et al., 2016]. For optimal quality of care the panel of essential diagnostic tests should be available at every DSD expert center.

An inventory of laboratories eligible for the ERN accreditation will be made in the first year of the Endo-ERN as task of the work-plan "Diagnostics \& Laboratory Analysis." If DSD centers are identified that lack appropriate laboratory facilities, an effort will have to be made to help establish these facilities or else referral pathways to other DSD centers will have to be created to ensure that individuals throughout Europe have access to high-quality diagnostics and consequently high-quality care. Another barrier may lie in the reimbursement of laboratory investigations. It may be challenging to solve problems of availability and reimbursement of laboratory diagnostics given the differences in health care systems and health insurance between European countries, and this requires political action.

\section{Novel Tests}

In addition to a defined set of laboratory investigations considered essential to the diagnostic process and monitoring of treatment in individuals with (suspected) DSD, other (novel) tests may be helpful in specific cases. For example, measurement of autoantibodies against steroidproducing cells may help to identify autoimmunity as a cause of ovarian failure [La Marca et al., 2010]. A 24-h urinary steroid profile may help distinguish between a rare adrenal enzyme deficiency such as apparent cortisone reductase deficiency and the more common 21-hydroxylase deficiency [Biason-Lauber et al., 2000]. Although currently mainly used in research, future clinical applications may be identified for insulin-like factor 3 (INSL3), a peptide hormone produced by Leydig cells which is thought to reflect their differentiation status and function [Ferlin et al., 2006]. Another example might be a common investigation such as measurement of steroids but in a different matrix, such as in scalp hair. Steroid levels measured using this noninvasive method reflect average serum levels over a longer period of time. Recently, measurement of androstenedione and 17OHP in scalp hair was suggested for monitoring treatment of $\mathrm{CAH}$ [Noppe et al., 2016].

Some novel tests are currently research-based but may possibly be useful for diagnosis or management in the future. An example is the assay that uses DHT-dependent transcriptional induction of the androgen receptor (AR) target gene apolipoprotein $\mathrm{D}$ in genital skin fibroblasts to assess androgen action [Hornig et al., 2016]. This assay is able to discriminate between individuals with androgen insensitivity caused by an $A R$ mutation and unaffected individuals. In a group of individuals with 46,XY DSD in whom androgen insensitivity was suspected but no $A R$ mutation was identified, the assay indicated disrupted androgen signaling in 37\% [Hornig et al., 2016]. This assay may be clinically useful for diagnostic as well as prognos- 
tic purposes, for example to predict the response to androgen treatment [Hornig et al., 2016].

In order to improve access to such tests, the ERN could create a web-based searchable database of available tests and which centers provide them. Such databases already exist for genetic tests on the orphanet website (www.orpha.net). Insight in who offers what tests will facilitate health care providers looking for specific tests outside their own center. This should also help to coordinate the development of (novel) tests. To efficiently make use of limited resources, work on novel tests should ideally be a joint action of the various DSD centers.

In conclusion, collaboration of clinicians and laboratory specialists is required to achieve harmonization of various laboratory tests, including standardization of protocols. There is a demand for European investments to establish a network of highly specialized endocrine reference laboratories and to create a web-based searchable database of available tests to achieve a Pan-European landscape ensuring access to optimal laboratory assessment for DSD. The establishment of this laboratory network should be embedded in a network of internationally accredited DSD expert centers.

\section{Disclosure Statement}

The authors have no conflicts of interest to declare.

\section{References}

Achermann JC, Ito M, Ito M, Hindmarsh PC, Jameson JL: A mutation in the gene encoding steroidogenic factor- 1 causes XY sex reversal and adrenal failure in humans. Nat Genet 22: 125-126 (1999).

Ahmed SF, Achermann JC, Arlt W, Balen AH, Conway $\mathrm{G}$, et al: UK guidance on the initial evaluation of an infant or an adolescent with a suspected disorder of sex development. Clin Endocrinol (Oxf) 75:12-26 (2011).

Ahmed SF, Achermann JC, Arlt W, Balen A, Conway $G$, et al: Society for Endocrinology UK guidance on the initial evaluation of an infant or an adolescent with a suspected disorder of sex development (Revised 2015). Clin Endocrinol (Oxf) 84:771-788 (2016).

Bashamboo A, Ferraz-de-Souza B, Lourenco D, Lin L, Sebire NJ, et al: Human male infertility associated with mutations in NR5A1 encoding steroidogenic factor 1 . Am J Hum Genet 87:505-512 (2010).

Bhasin S, Cunningham GR, Hayes FJ, Matsumoto AM, Snyder PJ, et al: Testosterone therapy in men with androgen deficiency syndromes: an Endocrine Society clinical practice guideline. J Clin Endocrinol Metab 95:2536-2559 (2010).

Bhowmick SK, Gidvani VK: Pitfalls of conventional human chorionic gonadotropin stimulation test to detect hormonally functional cryptorchid testes in midchildhood. Endocr Pract 6:8-12 (2000).

Biason-Lauber A, Suter SL, Shackleton CH, Zachmann M: Apparent cortisone reductase deficiency: a rare cause of hyperandrogenemia and hypercortisolism. Horm Res 53:260-266 (2000).
Ferlin A, Garolla A, Rigon F, Rasi Caldogno L, Lenzi A, Foresta C: Changes in serum insulinlike factor 3 during normal male puberty. J Clin Endocrinol Metab 91:3426-3431 (2006).

Feyaerts A, Forest MG, Morel Y, Mure PY, MorelJournel N, et al: Endocrine screening in 32 consecutive patients with hypospadias. J Urol 168:720-725 (2002).

Greaves RF, Jolly L, Hartmann MF, Ho CS, Kam RK, et al: Harmonisation of serum dihydrotestosterone analysis: establishment of an external quality assurance program. Clin Chem Lab Med 55:522-529 (2017).

Hornig NC, Ukat M, Schweikert HU, Hiort O, Werner R, et al: Identification of an AR mutation-negative class of androgen insensitivity by determining endogenous AR activity. J Clin Endocrinol Metab 101:4468-4477 (2016).

Hughes IA, Houk C, Ahmed SF, Lee PA, Group LC, Group EC: Consensus statement on management of intersex disorders. Arch Dis Child 91:554-563 (2006).

Kolon TF, Miller OF: Comparison of single versus multiple dose regimens for the human chorionic gonadotropin stimulatory test. J Urol 166:1451-1454 (2001).

Kuiri-Hanninen T, Sankilampi U, Dunkel L: Activation of the hypothalamic-pituitary-gonadal axis in infancy: minipuberty. Horm Res Paediatr 82:73-80 (2014).

Kulle A, Krone N, Holterhus PM, Schuler G, Greaves RF, Juul A, et al: Steroid hormone analysis in diagnosis and treatment of DSD: position paper of EU COST Action BM 1303 'DSDnet'. Eur J Endocrinol 176:P1-P9 (2017).

Kyriakou A, Dessens A, Bryce J, Iotova V, Juul A, et al: Current models of care for disorders of sex development - results from an international survey of specialist centres. Orphanet J Rare Dis 11:155 (2016).
La Marca A, Brozzetti A, Sighinolfi G, Marzotti S, Volpe A, Falorni A: Primary ovarian insufficiency: autoimmune causes. Curr Opin Obstet Gynecol 22:277-282 (2010).

Middle JG: Dehydroepiandrostenedione sulphate interferes in many direct immunoassays for testosterone. Ann Clin Biochem 44:173-177 (2007).

Noppe G, de Rijke YB, Koper JW, van Rossum EF, van den Akker EL: Scalp hair 17-hydroxyprogesterone and androstenedione as a longterm therapy monitoring tool in congenital adrenal hyperplasia. Clin Endocrinol (Oxf) 85:522-527 (2016).

Speiser PW, Azziz R, Baskin LS, Ghizzoni L, Hensle TW, et al: Congenital adrenal hyperplasia due to steroid 21-hydroxylase deficiency: an Endocrine Society clinical practice guideline. J Clin Endocrinol Metab 95:41334160 (2010).

Warner MH, Kane JW, Atkin SL, Kilpatrick ES: Dehydroepiandrosterone sulphate interferes with the Abbott Architect direct immunoassay for testosterone. Ann Clin Biochem 43: 196-199 (2006).

Wong T, Shackleton CH, Covey TR, Ellis G: Identification of the steroids in neonatal plasma that interfere with 17 alpha-hydroxyprogesterone radioimmunoassays. Clin Chem 38: 1830-1837 (1992).

Wudy SA, Wachter UA, Homoki J, Teller WM: 17 alpha-hydroxyprogesterone, 4-androstenedione, and testosterone profiled by routine stable isotope dilution/gas chromatographymass spectrometry in plasma of children. Pediatr Res 38:76-80 (1995). 